\section{Substanz P}

\author{
A. M. Gressner ${ }^{1}$ und O. A. Gressner ${ }^{2}$ \\ ${ }^{1}$ Labor Dr. Wisplinghoff Berlin, Berlin, Deutschland \\ ${ }^{2}$ Labor Dr. Wisplinghoff Köln, Köln, Deutschland
}

Englischer Begriff substance P ( $p=$ pain $)$

Definition Kurzkettiges, innerhalb von Nervenzellen und spezifischen endokrinen Zellen des Gastrointestinaltrakts im Körper weit verbreitetes Polypeptid mit Funktionen als sensorischer Neurotransmitter (von Schmerzreizen) und potenter Vasodilatator.

Beschreibung Das aus 11 Aminosäuren bestehende, als Präproprotein synthetisierte und proteolytisch prozessierte Peptid ist weit verbreitet im Gastrointestinaltrakt sowie im peripheren und zentralen Nervensystem, wo es als sensorischer Neurotransmitter, insbesondere bei Nozirezeptoren wirkt. Überträgt Schmerzreize (analgetischer Faktor), stimuliert glatte Muskelkontraktion im Gastrointestinaltrakt, Speichelsekretion, $>$ Histamin-Freisetzung, Vasodilatation und Superoxidproduktion in $>$ Makrophagen. Aufgrund ihrer Aminosäuresequenz H-Arg-Pro-Lys-Pro-Gln-Gln-Phe-Phe-GlyLeu-Met- $\mathrm{NH}_{2}$, die im Carboxy-terminalen Bereich mit der anderer amidierter Neuropeptide identisch ist (Phe-X-GlyLeu-Met- $\mathrm{NH}_{2}$ ), gehört Substanz P gemeinsam mit Neuromedin und Substanz K zur Familie der Tachykinine. Nüchternplasmakonzentration stark methodenabhängig, Richtwert $<240 \mathrm{ng} / \mathrm{L}$, Analyt instabil (eisgekühltes, Aprotinin-versetztes EDTAPlasma, was sofort tiefgefroren werden muss). Bestimmung mit $\triangleright$ Enzymimmunoassay (EIA) oder $\triangleright$ ChemolumineszenzAssay (CLIA).

Erhöhungen der Plasmakonzentration bei Karzinoidtumor, medullärem Schilddrüsenkarzinom und weiteren hormonaktiven gastrointestinalen Tumoren.

Selektive Katheterisierung zur Bestimmung von Substanz P dient der Lokalisationsdiagnostik des Karzinoidtumors.

\section{Literatur}

Severini C, Petrella C, Calissano P (2016) Substance P and Alzheimer's disease: emerging novel roles. Curr Alzheimer Res 13(9):964-972 